THE death is announced of Dr. Benedict Wallet Vilakazi, the eminent Zulu poet, who won a prize in the competition for literary works in African vernacular languages organized by the International African Institute in 1932 . He became assistant lecturer in Bantu languages in the University of Witwatersrand and was the first African in the Union to receive the degree of Doctor of Letters. At his funeral service Dr. C. Doke, his colleague and chief at the University, paid a notable tribute to his qualities. 'Vilakazi', he said, 'was obsessed with a great desire for the intellectual uplift of his people. He believed in a great and honourable future for the literature of the Bantu.... He has set before his people a great example.' We had the pleasure of printing in Africa his beautiful poem on the Victoria Falls.

\title{
A New Appointment
}

DR. S. F. NADEL, whose name and work are familiar to readers of this Journal, has been appointed Reader in Social Anthropology at Durham University. We hope to publish in our next number a review of his new book, The Nuba.

\section{Les Sciences humaines à Madagascar}

Nous sommes reconnaissants à M. J. Faublée pour la note suivante:

A Madagascar, l'organisation des recherches de sciences humaines commence seulement. Depuis la réunion de Madagascar à la France métropolitaine l'Académie malgache dut assurer un rôle de coordination. L'Académie est une réunion de malgachisants ayant publié au moins une étude sur un sujet malgache, choisis par cooptation. Elle publie un bulletin et des mémoires. Il est à noter que la plupart des membres sont européens. Des lettrés malgaches sont membres, mais publient rarement leurs travaux, interviennent peu dans les séances. Les malgachisants âgés qui dirigent la société sont remarquables par leur connaissance des anciennes coutumes. Ils s'intéressent à une ethnographie archaïque, évoquent les faits passés et l'ancienne société, négligènt de décrire l'évolution actuelle. La plupart demeurent à Tananarive; des régions échappent à toute étude.

La guerre empêcha de fonctionner normalement le service de la recherche scientifique. Monsieur Decary, qui le dirigeait, dut se limiter à quelques enquêtes, à coordonner les efforts des missions spécialisées envoyées de la métropole et ceux des chercheurs demeurant sur place. Son ambition était d'obtenir des monographies tribales homogènes et comparables. Sur les dix-huit tribus de Madagascar quatre groupes du Sud seulement ont fait l'objet de monographies assez détaillées. Au cours d'un séjour à Madagascar, de 1938 à I94I, je m'aperçus des difficultés de l'établissement de monographies tribales. Il y a trop peu de missions d'ethnographes professionnels pour les faire. Ceux qui résident sur place sont trop souvent limités à un domaine géographique trop restreint à l'intérieur d'une tribu. Pour eux une tribu est un domaine trop vaste, tandis qu'ils sont les mieux placés pour établir des monographies de villages. D'autre part la monographie a des défauts d'exposition: elle place sur des plans équivalents des faits d'importance inégale. Des techniques secondaires comme le lavage du linge, par exemple, prennent une importance trop grande.

Monsieur Decary organisa le dépouillement des derniers recensements. Il en résulta un travail important qu'il publia en collaboration avec Monsieur Castel: Modalités et conséquences des migrations intérieures récentes des populations malgaches. Cet ouvrage complète une carte ethnographique et démographique. Ces travaux mettent en valeur le fait capital de l'ethnographie de Madagascar: la population est tellement mêlée par les migrations que les anciens cadres tribaux ne peuvent servir de limites à un travail. Il est maintenant impossible 